ARTICLE

Received 11 Feb 2014 | Accepted 23 Apr 2014 | Published 29 May 2014

DOI: $10.1038 /$ ncomms4936

OPEN

\title{
Separating NADH and NADPH fluorescence in live cells and tissues using FLIM
}

\author{
Thomas S. Blacker ${ }^{1,2,3}$, Zoe F. Mann ${ }^{2,4}$, Jonathan E. Gale ${ }^{2,4}$, Mathias Ziegler ${ }^{5}$, Angus J. Bain ${ }^{3}$, \\ Gyorgy Szabadkai ${ }^{2,6, \star} \&$ Michael R. Duchen ${ }^{2, \star}$
}

NAD is a key determinant of cellular energy metabolism. In contrast, its phosphorylated form, NADP, plays a central role in biosynthetic pathways and antioxidant defence. The reduced forms of both pyridine nucleotides are fluorescent in living cells but they cannot be distinguished, as they are spectrally identical. Here, using genetic and pharmacological approaches to perturb $\mathrm{NAD}(\mathrm{P}) \mathrm{H}$ metabolism, we find that fluorescence lifetime imaging (FLIM) differentiates quantitatively between the two cofactors. Systematic manipulations to change the balance between oxidative and glycolytic metabolism suggest that these states do not directly impact $\mathrm{NAD}(\mathrm{P}) \mathrm{H}$ fluorescence decay rates. The lifetime changes observed in cancers thus likely reflect shifts in the NADPH/NADH balance. Using a mathematical model, we use these experimental data to quantify the relative levels of NADH and NADPH in different cell types of a complex tissue, the mammalian cochlea. This reveals NADPH-enriched populations of cells, raising questions about their distinct metabolic roles.

\footnotetext{
${ }^{1}$ Centre for Mathematics and Physics in the Life Sciences and Experimental Biology, University College London, London WC1E 6BT, UK. ${ }^{2}$ Research Department of Cell \& Developmental Biology, University College London, London WC1E 6BT, UK. ${ }^{3}$ Department of Physics and Astronomy, University College London, London WC1E 6BT, UK. ${ }^{4}$ UCL Ear Institute, University College London, London WC1X 8EE, UK. ${ }^{5}$ Department of Molecular Biology, University of Bergen, N-5008 Bergen, Norway. ${ }^{6}$ Department of Biomedical Sciences, University of Padua and CNR Neuroscience Institute, Padua 35121, Italy. ${ }^{\star}$ These authors contributed equally to this work. Correspondence and requests for materials should be addressed to M.R.D. (email: m.duchen@ucl.ac.uk).
} 
T he cellular redox state is the central regulator of energy production and intermediary metabolism, playing a crucial role in health and disease ${ }^{1}$. The nicotinamide adenine dinucleotide $\left(\mathrm{NAD}^{+} / \mathrm{NADH}\right)$ and nicotinamide adenine dinucleotide phosphate $\left(\mathrm{NADP}^{+} / \mathrm{NADPH}\right)$ redox couples are the major determinants of redox state in the cell. However, these engage in distinct metabolic pathways. NAD drives ATP production in the cytosol by glycolysis and in the mitochondria by oxidative phosphorylation, while the phosphorylated analogue NADP governs lipid, amino acid and nucleotide biosynthetic pathways and the defence against reactive oxygen species by glutathione $(\mathrm{GSH})^{2}$. Free radical generation is therefore determined by the redox state of NAD, while NADP redox state is key to antioxidant defence ${ }^{3}$. The relative abundance of the two pyridine nucleotides and their redox balance thus mediates cell fate in a wide range of diseases, including cancer, diabetes and neurodegeneration. Quantifying their behaviour is therefore essential in understanding the role of metabolism in these diseases. However, separating the contributions of the two pools in intact tissues has proven technically challenging ${ }^{4}$.

In the 1960s, Britton Chance et al. ${ }^{5}$ showed that live tissues illuminated with ultraviolet light emit blue fluorescence, arising primarily from mitochondrial NADH. The nicotinamide moiety of NADH absorbs light of wavelength $340 \pm 30 \mathrm{~nm}$ and emits fluorescence at $460 \pm 50 \mathrm{~nm}$. As NADP is phosphorylated at a remote site of the molecule, the fluorescence properties of the nicotinamide ring of NADPH are identical to those of $\mathrm{NADH}^{6,7}$. Thus, changes in autofluorescence intensity may reflect changes in either [NADH] or [NADPH], often denoted as $\mathrm{NAD}(\mathrm{P}) \mathrm{H}$ to indicate the uncertain origin of the signal ${ }^{8,9}$.

Fluorescence lifetime imaging (FLIM) allows the study of $\mathrm{NAD}(\mathrm{P}) \mathrm{H}$ photochemistry inside living tissue $\mathrm{e}^{10-12}$. This technique measures the rates of fluorescence decay of $\mathrm{NAD}(\mathrm{P}) \mathrm{H}$, an excited-state process occurring over nanosecond time scales, which is highly sensitive to the immediate environment of the fluorophore. We have therefore investigated whether the fluorescence lifetime of cellular NADPH differs from that of cellular NADH, reflecting the different set of enzymes to which the two cofactors bind. Indeed, a substantial literature documents variations in the fluorescence lifetime of $\mathrm{NAD}(\mathrm{P}) \mathrm{H}$ in a range of physiological and pathological conditions ${ }^{13-18}$. However, despite the potential clinical applications of autofluorescence lifetime measurements, such as delineating the boundaries of accessible cancers ${ }^{14}$, the biochemical basis for these variations remains unknown. Moreover, the issue of whether FLIM may permit separation of NADH and NADPH fluorescence to resolve cellular specializations and dynamics in intact tissues is yet to be addressed. In the present study, we have explored the fluorescence decay properties of NADH and $\mathrm{NADPH}$ in living cells and tissues. Combined with computational and mathematical modelling, we have found that $\mathrm{NAD}(\mathrm{P}) \mathrm{H}$ fluorescence lifetime characteristics discriminate between NADH and NADPH. This provides a unique approach to identify cells within complex tissues that are enriched in NADPH, thus raising questions about their metabolic roles and specialization. We have also analysed the impact of altered metabolic state on $\mathrm{NAD}(\mathrm{P}) \mathrm{H}$ fluorescence decay characteristics, helping to place changes in lifetimes observed in transformed neoplastic cells on a firm biochemical footing.

\section{Results}

$\mathrm{NAD}(\mathrm{P}) \mathrm{H}$ fluorescence decay reflects bound NADPH/NADH ratio. We have previously shown that the fluorescence lifetime of NADPH is identical to that of NADH in solution ${ }^{19}$, demonstrating that fluorescence from the free, unbound pyridine nucleotides cannot be discriminated on the basis of their fluorescence lifetime. However, it has long been known that this value increases from $0.3-0.8$ to $1-6.5 \mathrm{~ns}$ on binding to an enzyme, depending on the target to which the cofactor binds ${ }^{10,20}$, as well as by the simultaneous presence of substrate molecules ${ }^{21}$. Canonical NAD(P)H FLIM studies in live cells and tissues resolve two fluorescence lifetimes at each pixel; one of the order of $0.4 \mathrm{~ns}$ and the other larger and more variable at $\sim 2 \mathrm{~ns}$ or more $\mathrm{r}^{11,12,22}$. These represent the freely diffusing $\left(\tau_{\text {free }}\right)$ and enzyme-bound $\mathrm{NAD}(\mathrm{P}) \mathrm{H}\left(\tau_{\text {bound }}\right)$ pools, respectively, confirmed by timeresolved anisotropy imaging studies ${ }^{23}$. As the distribution of bound $\mathrm{NAD}(\mathrm{P}) \mathrm{H}$ species will inevitably be more heterogeneous than implied by the single long lifetime, we suggest that $\tau_{\text {bound }}$ is a weighted mean of the fluorescence lifetimes of enzyme-bound species, a conclusion supported by computational modelling (see Supplementary Fig. 1a-m and Supplementary Note 1). It is primarily this parameter that has previously been observed to vary with changes in metabolism ${ }^{11,24-26}$.

Next, to understand the effect of varying [NADPH]/[NADH] ratios on $\tau_{\text {bound }}$, we acquired FLIM images of $\mathrm{NAD}(\mathrm{P}) \mathrm{H}$ in live HEK293 cells in which NADPH levels were genetically and pharmacologically manipulated. In wild-type cells, the fluorescence decays obtained at each pixel required a two-component model to yield good fits (Fig. la,b), as expected, with $\chi_{r}^{2}$ values close to unity. As such, addition of further components was not appropriate, to avoid inaccurate weightings due to overfitting ${ }^{27}$. Identical fluorescence decay parameters were observed in the cytosol and mitochondria, with mean ( \pm s.d.) values of $\tau_{\text {bound }}=2.7 \pm 0.2 \mathrm{~ns}, \quad \alpha_{\text {bound }}=0.19 \pm 0.01 \quad$ (fraction of bound $\mathrm{NAD}(\mathrm{P}) \mathrm{H}$ ) and $\tau_{\text {free }}=0.36 \pm 0.04 \mathrm{~ns}$ (Supplementary Table 1 ). Interestingly, $\tau_{\text {bound }}$ was significantly smaller in the nucleus at $2.3 \pm 0.2 \mathrm{~ns} \quad(P=1 \mathrm{E}-8$, two-tailed Student's $t$-test, $n=17$ images).

To explain reported variations in $\tau_{\text {bound }}$, we hypothesized that, since NADH and NADPH are associated with different binding site structures inside the cell ${ }^{28}$, the measured $\tau_{\text {bound }}$ value could reflect the proportion of the two cofactors present. We therefore measured NAD(P)H fluorescence lifetimes in HEK293 cell lines in which $\mathrm{NAD}^{+}$kinase (NADK) was either overexpressed $(\mathrm{NADK}+)$ or knocked down $(\mathrm{NADK}-)^{29}$. NADK is the key determinant of NADPH concentration inside the cell, and its overexpression results in a 10- to 15 -fold higher [NADPH] in $\mathrm{NADK}+$ relative to NADK - cells, leaving [NADH] relatively unaffected $^{29}$. This was reflected by an NAD $(\mathrm{P}) \mathrm{H}$ fluorescence intensity $\sim 10$-fold brighter in NADK + compared with NADK - cells (Supplementary Fig. 2b). FLIM revealed that $\tau_{\text {bound }}$ was significantly larger in the NADK + cells ( $\tau_{\text {bound }}=3.6 \pm 0.2 \mathrm{~ns}$ in the mitochondria, $3.8 \pm 0.2 \mathrm{~ns}$ in the cytosol) compared with the NADK - cells $\left(\tau_{\text {bound }}=2.7 \pm 0.1 \mathrm{~ns}\right.$ in both subcellular regions, $P=1 \mathrm{E}-11$ and $P=5 \mathrm{E}-12$, two-tailed Student's $t$-test, $n=9$, Fig. $1 \mathrm{c}$ and Supplementary Table 1).

These data suggest that increased concentrations of bound NADPH result in increased values of $\tau_{\text {bound. }}$ To verify this interpretation, we explored the impact of epigallocatechin gallate (EGCG) on the NAD(P)H FLIM parameters. EGCG is a potent competitive inhibitor of NADPH binding ${ }^{30}$ with no effect on NADH binding indicated by the BRENDA database ${ }^{31}$. Preferential competition for NADPH-binding sites by EGCG would decrease the bound NADPH population, leaving the bound NADH population unaffected. The value of $\alpha_{\mathrm{bound}}$ should therefore be more sensitive to EGCG treatment in NADK + cells than in NADK - cells, as bound NADPH will form a greater fraction of the total population of enzyme-bound $\mathrm{NAD}(\mathrm{P}) \mathrm{H}$ species. As predicted, $\alpha_{\text {bound }}$ decreased significantly from $0.18 \pm 0.02$ to $0.13 \pm 0.02$ in both the mitochondria and cytosol 
$(P=4 \mathrm{E}-10$, two-tailed Student's $t$-test, $n=9)$ on EGCG treatment in NADK + cells but remained constant in NADK cells, confirming the NADPH specificity of this compound (see Fig. 1d). Exposure to EGCG decreased $\tau_{\text {bound }}$ to $3.1 \pm 0.2 \mathrm{~ns}$ in both the mitochondria and cytosol of NADK + cells $(P=2 \mathrm{E}-12$, two-tailed Student's $t$-test, $n=9)$ and to $2.5 \pm 0.2 \mathrm{~ns}$ in their nuclei $(P=3 \mathrm{E}-7$, two-tailed Student's $t$-test, $n=9)$, and did not significantly affect NADK - cells (Fig. 1c). In addition, treatment with this compound did not affect $\tau_{\text {free }}$ (Supplementary Fig. 2c,d). Thus, these data are consistent with a model where $\tau_{\text {bound }}$ reports the amount of enzyme-bound NADPH relative to bound NADH.

These data suggest that $\tau_{\text {bound }}$ can be used to quantify enzymebound NADPH/NADH ratios. We therefore developed a numerical model to quantify this phenomenon and generate predictions (see Supplementary Fig. 1k-o and Supplementary Note 2). By combining the enzyme-bound NAD(P)H fluorescence lifetimes measured in the NADK + and NADK - cells, the biochemically quantified $[\mathrm{NADH}]$ and $[\mathrm{NADPH}]$ values in each cell line $\mathrm{e}^{29}$ and a mathematical model in which NADH and $\mathrm{NADPH}$ were assumed to possess discrete and distinct fluorescence lifetimes when bound inside the cell, we found that $\tau_{\text {bound }}$ would describe the $[\mathrm{NADPH}] /[\mathrm{NADH}]$ ratio by

$$
\frac{[\mathrm{NADPH}]}{[\mathrm{NADH}]}=\frac{\tau_{\text {bound }}(\mathrm{ns})-1.5}{4.4-\tau_{\text {bound }}(\mathrm{ns})}
$$

Application of this model to the NADK + and NADK - data (see Supplementary Note 3 ) showed that the concentration of bound NADH remained constant on EGCG application, while the concentration of bound NADPH decreased $\sim 3$-fold with small differences in each subcellular compartment, supporting our hypothesis.

Lifetime changes reflect mechanism of metabolic perturbation. In previous work ${ }^{11}$, shortening of $\tau_{\text {bound }}$ observed in tumours has been attributed to a shift from oxidative to glycolytic metabolism which occurs in many cancers, the so-called Warburg effect. To investigate this hypothesis in the light of the results described here, FLIM images of wild-type HEK293 cells were acquired following a range of manipulations that alter the balance of ATP production by aerobic or anaerobic pathways. Dependence on glycolysis was achieved by inhibition of mitochondrial oxidative phosphorylation using rotenone $(10 \mu \mathrm{M})$ or uncoupling using
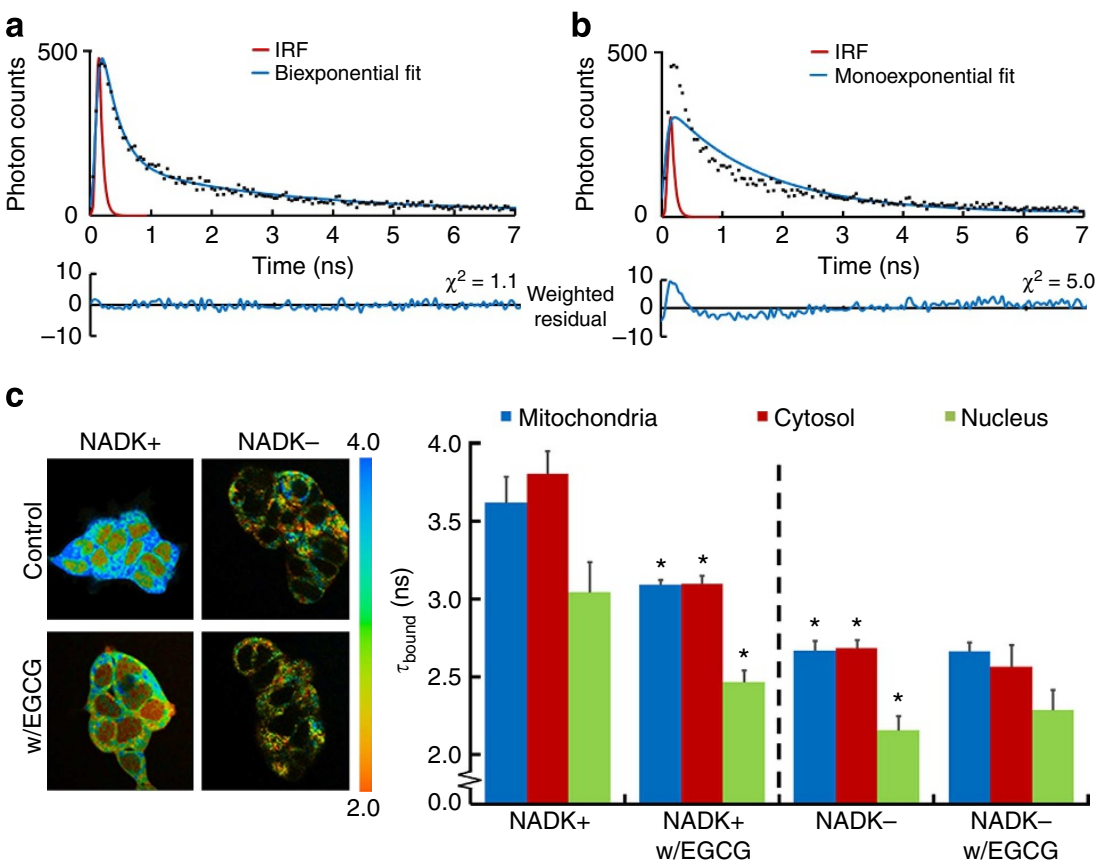

d
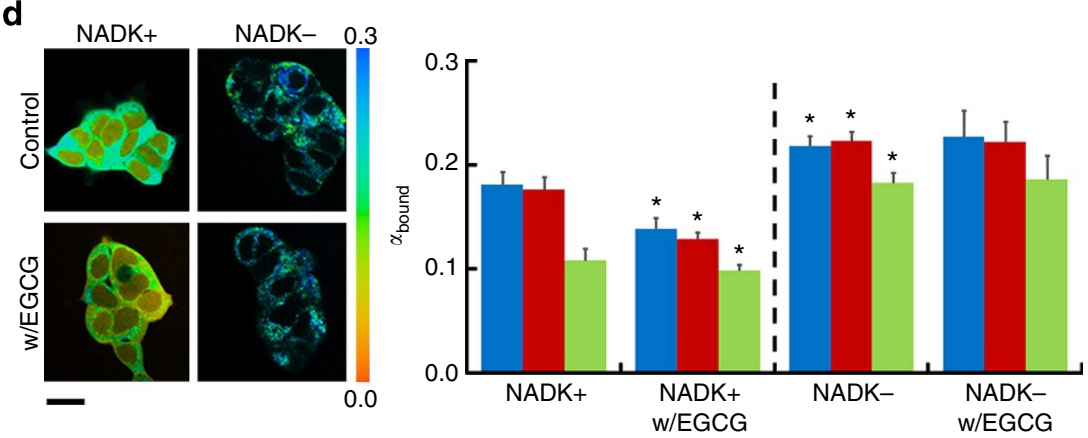

Figure 1 | $\tau_{\text {bound }}$ reflects the enzyme-bound NADPH/NADH ratio in intact cells. (a,b) A biexponential decay model adequately described the NAD(P)H fluorescence decay measured in wtHEK293 cells (IRF, instrument response function). The mean $\chi_{r}^{2}$ was $1.24 \pm 0.08$ compared with $4 \pm 1$ with a monoexponential fit (representative data from $n=17$ experiments). (c,d) Representative colour-coded images and mean $\tau_{\text {bound }}$ and $\alpha_{\text {bound }}$ values in NADK + and NADK - HEK293 cells prior and following treatment with EGCG $(100 \mu M)$, a competitive inhibitor of NADPH binding. Scale bar, $20 \mu$ m. Error bars indicate \pm s.d., ${ }^{\star} P<0.05$ (two-tailed Student's $t$-test, $n=9$ ). 
FCCP $(1 \mu \mathrm{M})$. The cells were driven to a more oxidative phenotype by inhibition of glycolysis by glucose deprivation in the presence of deoxyglucose $(10 \mathrm{mM})$, while pyruvate $(1 \mathrm{mM})$ or lactate $(10 \mathrm{mM})$ were provided as mitochondrial-specific substrates.

Ultraviolet confocal microscopy was used to establish the time taken for the redox state of the $\operatorname{NAD}(\mathrm{P})$ pool to reach a new steady state following each treatment (Fig. 2a-d). Inhibition of glycolysis decreased the cellular $\mathrm{NAD}(\mathrm{P}) \mathrm{H}$ fluorescence intensity by $26 \pm 6$ and $22 \pm 8 \%$ with pyruvate and lactate supplied, respectively. Rotenone increased steady-state $\mathrm{NAD}(\mathrm{P}) \mathrm{H}$ fluorescence intensity by $20 \pm 5 \%$ while FCCP caused a decrease by $38 \pm 2 \%$ (each $n=3$ ). FLIM images were acquired at the steadystate fluorescence intensity levels following each treatment (Fig. $2 \mathrm{e}-\mathrm{g}$ and Supplementary Table 2 ). Rotenone caused $\tau_{\text {bound }}$ to decrease significantly from $2.7 \pm 0.2$ to $2.52 \pm 0.05 \mathrm{~ns}$ in both the mitochondria and cytosol $(P=6 \mathrm{E}-4$, two-tailed Student's $t$-test, $n=9$ ), suggesting that this treatment caused the concentration of $\mathrm{NADH}$ present in the cell to increase relative to the concentration of NADPH, following inhibition of $\mathrm{NADH}$ oxidation by complex I. In contrast, $\tau_{\text {bound }}$ did not change in response to FCCP $(P>0.05$, two-tailed Student's $t$-test, $n=13)$. This lack of change in $\tau_{\text {bound }}$ suggested that uncoupling caused the oxidation of both NAD and NADP pools in equal measure. Increased oxidation of the NAD pool was to be expected on uncoupling due to the increased complex I activity, and the equal oxidation of the NADP pool was likely caused by the action of the mitochondrial transhydrogenase. In respiring mitochondria, this inner mitochondrial membrane protein transfers hydride from $\mathrm{NADH}$ to $\mathrm{NADP}^{+}$powered by translocation of protons from the intermembrane space to the mitochondrial matrix ${ }^{32}$. However, on uncoupling, NADPH is oxidized and the hydride is passed to
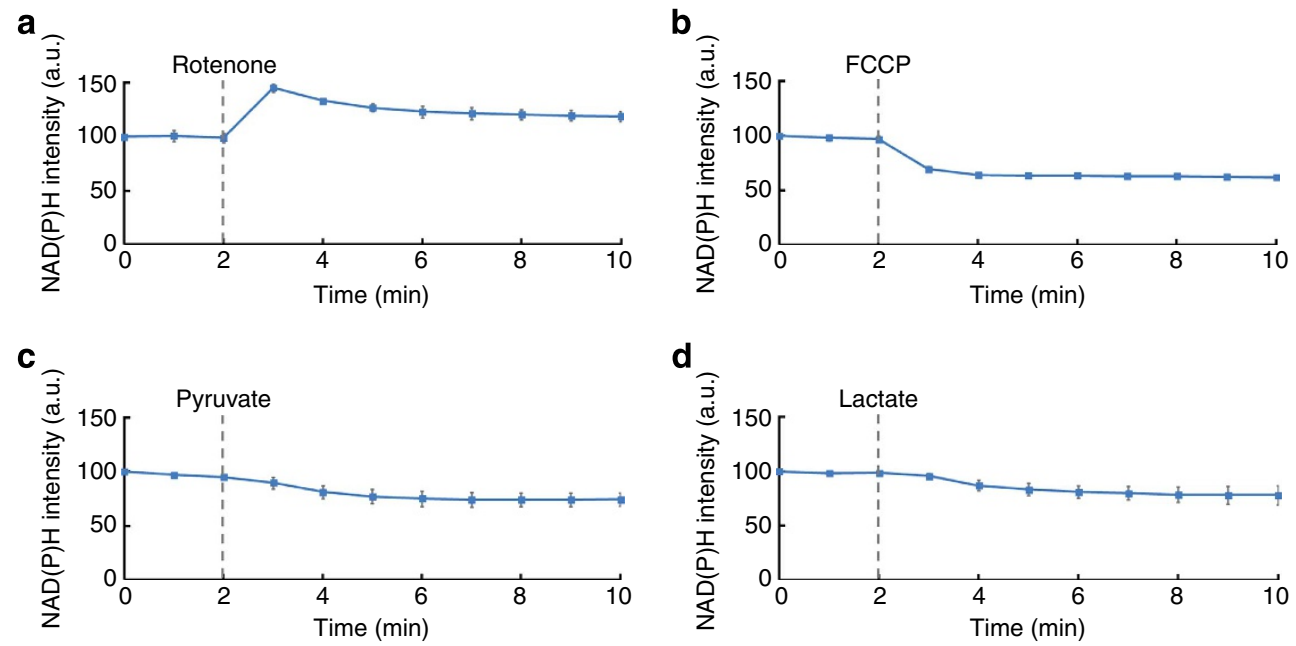

e
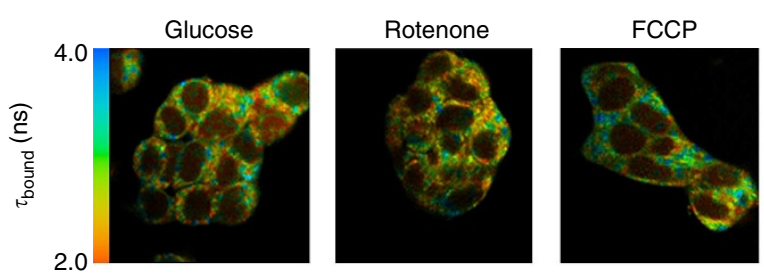

(Glucose deprivation)
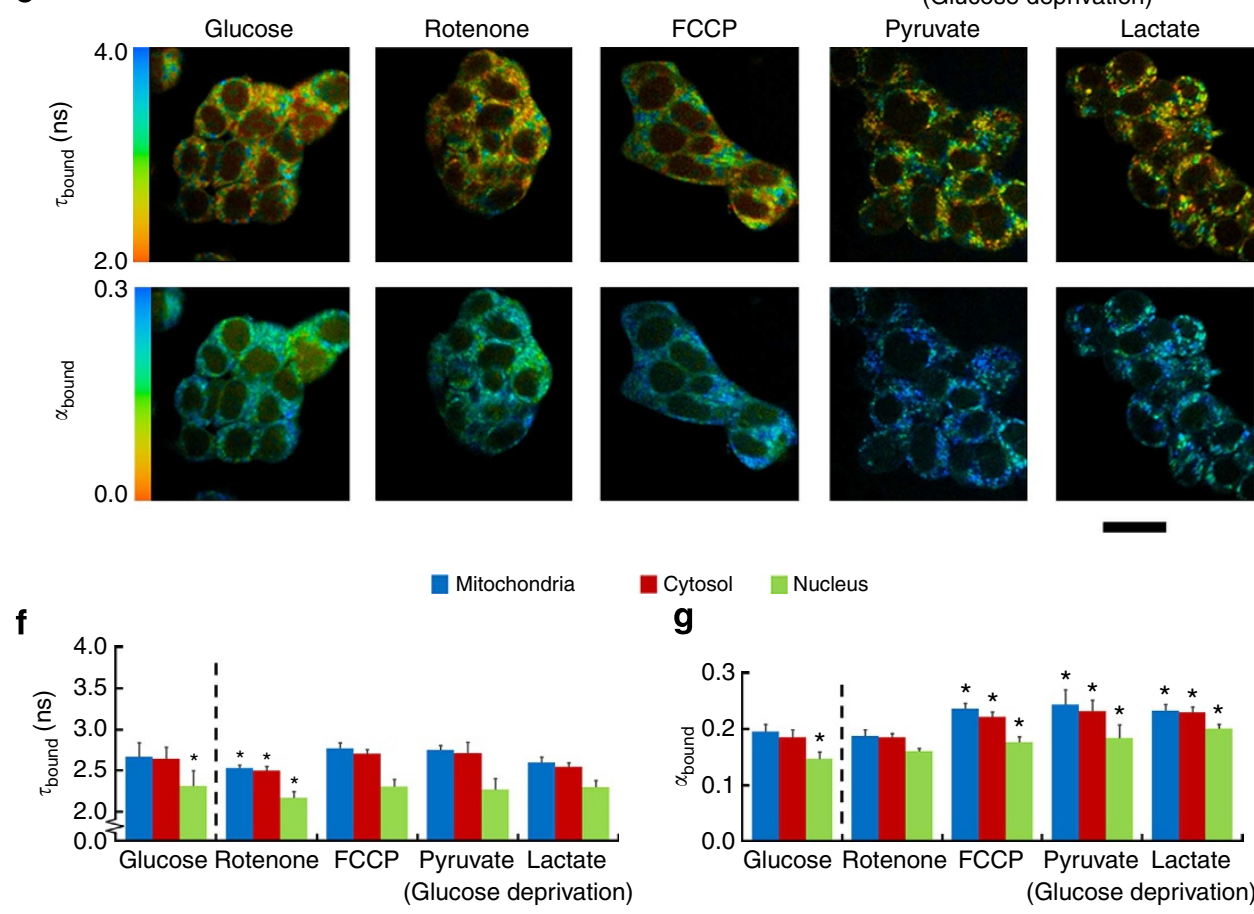

Cytosol Nucleus

g

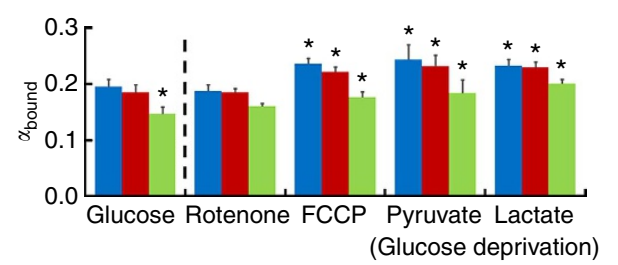

Figure 2 | NAD(P)H fluorescence decay responses to metabolic perturbation are mechanism dependent. (a-d) Time series of NAD(P)H fluorescence intensity following treatments chosen to perturb oxidative (a: respiratory chain inhibition by rotenone, $\mathbf{b}$ : uncoupling with FCCP) and glycolytic metabolism (glucose replaced by deoxyglucose with $\mathbf{c}$ : pyruvate or $\mathbf{d}$ : lactate supplied as substrate). (e) Colour-coded images and (f,g) quantification of changes in $\tau_{\text {bound }}$ and $\alpha_{\text {bound }}$ on application of treatment. Scale bar, $20 \mu \mathrm{m}$. Error bars indicate \pm s.d., ${ }^{\star} P<0.05$ (two-tailed Student's $t$-test, $n=9$ ). 
$\mathrm{NAD}^{+}$to produce $\mathrm{NADH}^{33}$. Relative to controls, glucose deprivation in the presence of either pyruvate or lactate supply failed to significantly alter $\tau_{\text {bound }}(P>0.05$, two-tailed Student's $t$-test, both $n=9)$. However, the mean value of $\tau_{\text {bound }}$ in both the mitochondria and cytosol of $2.70 \pm 0.01 \mathrm{~ns}$ in the presence of pyruvate was significantly larger than its value of $2.57 \pm 0.04 \mathrm{~ns}$ in the presence of lactate $(P=7 \mathrm{E}-6$, two-tailed Student's $t$-test, $n=9)$. As lactate dehydrogenase promotes $\mathrm{NADH}$ production during the conversion of lactate to pyruvate, the significantly smaller $\tau_{\text {bound }}$ value in the presence of lactate relative to pyruvate supports the interpretation developed in this work that this parameter reflects $\mathrm{NADPH} / \mathrm{NADH}$ ratio, assuming $\mathrm{NADPH}$ production was identical in the presence of the two substrates.

While changes in $\tau_{\text {bound }}$, and thus the NADPH/NADH ratio, reflected the specific treatment causing a defect in OXPHOS or glycolysis, we observed that each treatment causing a net oxidation of the combined $\mathrm{NAD}(\mathrm{P})$ pools caused $\alpha_{\text {bound }}$ to increase (Fig. 2g). This is in support of previous suggestions that this parameter, reflecting the enzyme-bound population fraction of $\mathrm{NAD}(\mathrm{P}) \mathrm{H}$, reports acute changes in the redox state of the cell $^{25}$. However, confirming whether this parameter reflects phenotypic differences in the redox state of different cell types requires further investigation. Interestingly, each oxidizing treatment also caused an increase in $\tau_{\text {free }}$ (Supplementary Fig. 2e,f). However, as these lifetimes lie close to the time resolution of the FLIM system, such small differences may be an artefact of the fitting process, such as an interdependency between $\tau_{\text {free }}$ and $\alpha_{\text {bound }}$ (Supplementary Fig. 1j).

The lack of significant change in $\tau_{\text {bound }}$ in response to FCCP treatment or replacement of glucose with pyruvate or lactate, along with the very small, if significant, change in this parameter following rotenone treatment relative to the pathophysiological variations reported in the literature, suggests that changes in $\mathrm{NAD}(\mathrm{P}) \mathrm{H}$ fluorescence decay cannot be simply attributed to alterations in the balance between oxidative and glycolytic metabolism. Accordingly, no correlation between $\tau_{\text {bound }}$ and the balance of ATP production by glycolytic or oxidative means was observed by measuring the rates of oxygen consumption and lactate release in the wild-type, NADK + and NADK - HEK293 cell lines (see Supplementary Fig. 3 and Supplementary Tables 3 and 4). Altogether, these data strongly suggest that any variation in the $\mathrm{NAD}(\mathrm{P}) \mathrm{H}$ fluorescence decay parameter $\tau_{\text {bound }}$ will be specific to the mechanism of metabolic perturbation, such as mitochondrial dysfunction caused either by respiratory chain inhibition or uncoupling, or the utilization of different substrates following inhibition of glycolysis. Such specificity has the potential to aid the study of the variety of possible metabolic rearrangements that may occur in cancer and other pathologies.

FLIM reveals metabolic variations in complex tissues. The abundance of NADPH and NADH in live cells can be measured a

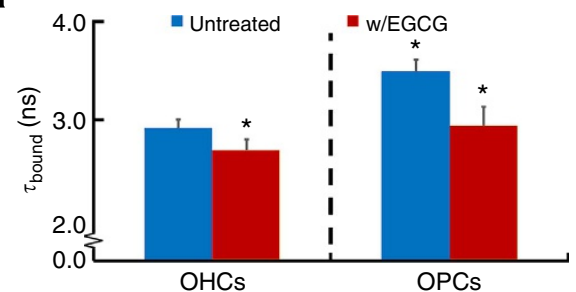

b

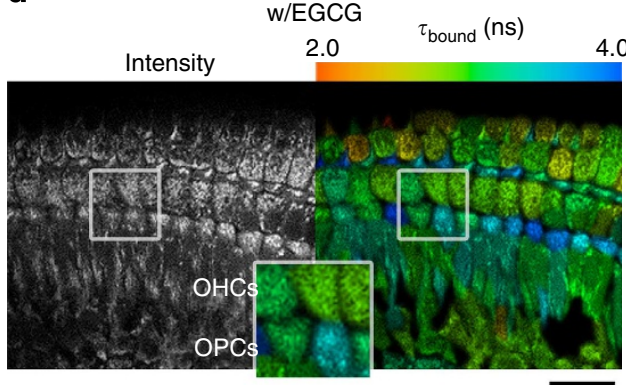

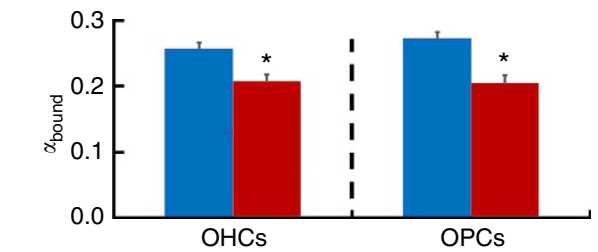

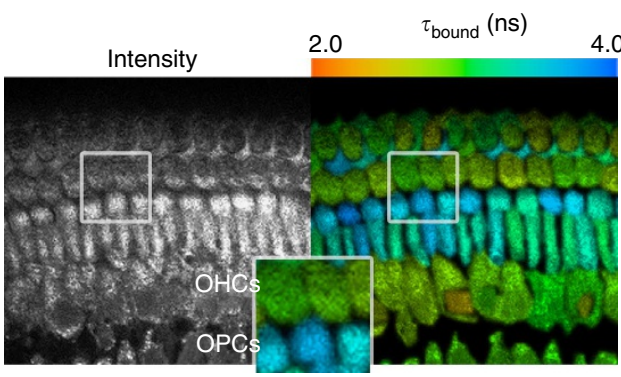

e

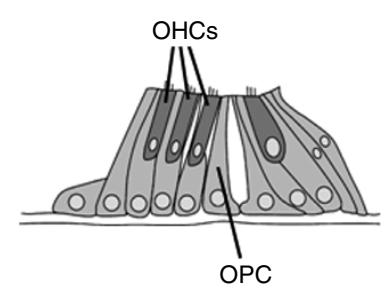

$\mathbf{f}$

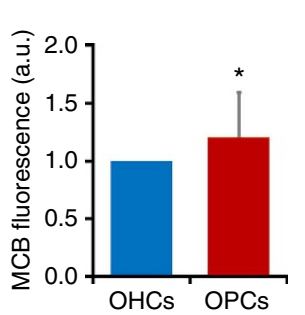

g

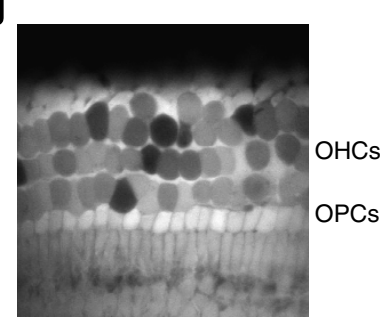

Figure 3 | Supporting cells in the mammalian cochlea exhibit increased enzyme-bound NADPH. (a) Mean $\tau_{\text {bound }}$ and (b) $\alpha_{\text {bound }}$ values in outer hair cells (OHC's) and adjacent outer pillar 'supporting' cells (OPC's) under control conditions and following application of EGCG (200 $\mu$ M). Error bars indicate \pm s.d., ${ }^{\star} P<0.05$ (two-tailed Student's $t$-test, $n=11$ ). (c,d) Corresponding representative FLIM images colour coded for the mean parameter value in each cell. Scale bar, $25 \mu \mathrm{m}$. (e) Schematic diagram showing organ of Corti in the cochlear explants, indicating the positions of OHC's and OPC's. (f) Mean fluorescence intensity in OPC's and OHC's. Error bars indicate \pm s.d., ${ }^{\star} P<0.05$ (Wilcoxon signed-rank test, $n=17$ ). (g) Representative image following MCB staining for GSH concentration. 
in tissues by biochemical means such as high performance liquid chromatography ${ }^{29,34}$. However, a microscopy-based imaging approach permits measurement of $\mathrm{NADH}$ and $\mathrm{NADPH}$ in discrete compartments within a cell or in different cell types within a complex tissue. Indeed, we have observed consistently smaller values of $\tau_{\text {bound }}$ in the nucleus compared with the rest of the cell. Combining enzyme localization data ${ }^{31}$ with the analysis and model provided here, NAD(P)H FLIM indicates that nuclear glucose metabolism favours NADH-producing glycolysis rather than NADPH-producing pentose phosphate pathway (PPP), resulting in a more oxidized NADP pool in this region (see Supplementary Note 4).

To explore the application of NAD(P)H FLIM in multicellular specimens, the redox metabolism of the mammalian cochlea was investigated. The cochlea is a structure containing a highly ordered system of functionally distinct cell types, which are easily identifiable from their architecture (Fig. 3e). FLIM imaging of cochlear explant cultures revealed that $\tau_{\text {bound }}$ differed markedly between different cell types. The glia-like outer pillar 'supporting' cells (OPCs) exhibited an extended fluorescence lifetime of $3.5 \pm 0.1 \mathrm{~ns}$, while the mean value of $\tau_{\text {bound }}$ measured in the neighbouring sensory receptors, the outer hair cells (OHCs), was significantly lower at $2.9 \pm 0.1 \mathrm{~ns}(P=2 \mathrm{E}-15$, two-tailed Student's $t$-test, $n=19$, Fig. 3a-d). EGCG treatment decreased $\tau_{\text {bound }}$ in supporting cells by $0.6 \pm 0.2 \mathrm{~ns}(P=1 \mathrm{E}-6$, two-tailed Student's $t$-test, $n=11$ ) to a mean of $2.9 \pm 0.2 \mathrm{~ns}$, but by only $0.2 \pm 0.2 \mathrm{~ns}(P=8 \mathrm{E}-4$, two-tailed Student's $t$-test, $n=11)$ to a mean of $2.7 \pm 0.1 \mathrm{~ns}$ in hair cells (Supplementary Table 5). These data confirm that the differences in fluorescence lifetime between cell types were a measure of differences in the bound $\mathrm{NADPH} / \mathrm{NADH}$ ratio.

Application of the mathematical model developed in this work (Supplementary equations 10 and 11) implied that the total concentration of reduced pyridine nucleotides were equal in the OHCs and OPCs. In the OHCs, the absolute concentrations of $\mathrm{NADH}$ and $\mathrm{NADPH}$ were equal at $1 \pm 0.2$ a.u. However, in the OPCs, the concentration of NADPH was significantly larger at $1.4 \pm 0.3$ a.u. $(P=4 \mathrm{E}-4$, Wilcoxon signed-rank test, $n=19)$, with a correspondingly smaller concentration of NADH $(0.7 \pm 0.2$ a.u., $P=2 \mathrm{E}-4$, Wilcoxon signed-rank test, $n=19$ ). To assess the functional significance of this finding, we measured the distribution of GSH in the tissue using monochlorobimane (MCB) staining, since a major demand for $\mathrm{NADPH}$ in glial cells arises from GSH turnover ${ }^{35}$. The glia-like OPCs showed significantly higher $[\mathrm{GSH}]$ compared with $\mathrm{OHCs}(P=0.02$, Wilcoxon signedrank test, $n=17$, Fig. 3f,g), consistent with a functional requirement for $\mathrm{NADPH}$ enrichment. Thus, our interpretation of $\tau_{\text {bound }}$ is consistent with alternative indicators of cellular redox state.

\section{Discussion}

This work shows that FLIM can be used effectively to differentiate between NADH and NADPH at the level of the single cell or organelle. The results suggest that enzyme-bound $\mathrm{NADPH}$ possesses a significantly larger fluorescence lifetime than enzyme-bound $\mathrm{NADH}$ within the cellular environment, so that the proportion of enzyme-bound NADPH and NADH present in live tissue determines the lifetime of their combined fluorescence decay. By making the simplifying assumption that bound NADH and bound NADPH possess finite and distinct fluorescence lifetimes inside the cell, the relative contribution of each cofactor to the combined fluorescence signal could be calculated. With excitation at $700 \mathrm{~nm}$ and a $435-485 \mathrm{~nm}$ detection window, the intracellular fluorescence lifetimes of NADH and NADPH were predicted to be $1.5 \pm 0.2$ and $4.4 \pm 0.2 \mathrm{~ns}$ respectively. It is reasonable to hypothesize that these conclusions can be extended to all cell types as the fluorescence lifetime of $\mathrm{NAD}(\mathrm{P}) \mathrm{H}$ when bound to an enzyme is determined by its local environment in the binding site ${ }^{21}$, and the NADH and NADPH-binding sites are two of the most highly conserved in all biology ${ }^{36,37}$. Indeed, we have observed values of $\tau_{\text {bound }}$ that are similar in magnitude across a range of cell types, including isolated ventricular cardiomyocytes and neurons in culture or in brain slices (data not shown).

The analysis and model presented herein are consistent with previously published NAD(P)H FLIM studies. In 2008, Niesner et $a .^{38}$ performed a novel study on the decay of $\mathrm{NAD}(\mathrm{P}) \mathrm{H}$ fluorescence in granulocytes in the presence of Aspergillus fumigatus fungus. The parameter $\tau_{\text {bound }}$ was $\sim 2 \mathrm{~ns}$ within the bulk cytosol of the granulocytes. However, localized subplasmalemmal regions of the cytosol in contact with the fungus displayed increased values of $3.7 \mathrm{~ns}$. This was attributed to a unique fluorescence lifetime of NADPH when bound to the $\mathrm{NADPH}$ oxidase activated in response to pathogenic exposure. However, the computational simulations performed here showed that $\tau_{\text {bound }}$ is a weighted average of the fluorescence lifetimes of the enzyme-bound $\mathrm{NAD}(\mathrm{P}) \mathrm{H}$ species present. For NADPH oxidase alone to cause an increase in $\tau_{\text {bound }}$ as large as that observed in the granulocytes would require this enzyme to be present at a greater concentration than the NADH-binding enzymes of the cytosol. The Model Organism Protein Expression Database (MOPED) $)^{39}$ within the GeneCards human gene compendium $^{40}$ shows that expression of glyceraldehyde 3-phosphate dehydrogenase outweighs that of the NADPHbinding subunit of NADPH oxidase (neutrophil cytosolic factor $2)^{41}$ by around 100 to one in neutrophils. It is therefore more likely that the $\mathrm{NAD}(\mathrm{P}) \mathrm{H}$ fluorescence lifetime observed in the regions of the cytosol where NADPH oxidase was activated was due to increased local NADPH production, as implied from the results reported here. Indeed, the large quantities of superoxide produced by NADPH oxidase requires plentiful supply of NADPH. Activation of this enzyme is thus associated with increased flux of glucose through the $\mathrm{PPP}^{42}$.

Changes in the fluorescence decay of $\mathrm{NAD}(\mathrm{P}) \mathrm{H}$ have recently been observed in applications ranging from wound healing ${ }^{17}$ to stem cell differentiation ${ }^{18}$ and necrotic deterioration of skin ${ }^{15}$ to staurosporine-induced apoptosis ${ }^{16}$. The large number of studies reporting differences between the fluorescence lifetime of $\mathrm{NAD}(\mathrm{P}) \mathrm{H}$ in healthy control cells and cells at different stages of carcinogenesis ${ }^{11,43,44}$ have prompted the design of clinical instruments for the detection, diagnosis and staging of accessible tumours using time-resolved autofluorescence measurements ${ }^{14,45-50}$. While the Warburg effect is the most well known of the metabolic shifts occurring during cancer development, tumorigenesis is also associated with variations in glucose flux through the PPP and various biosynthetic pathways utilizing $\mathrm{NADPH}^{51}$. As none of the severe pharmacological perturbations to cytosolic or mitochondrial ATP production applied in this work could reproduce responses in $\tau_{\text {bound }}$ of the magnitude caused by an increased NADPH/NADH ratio, our results strongly suggest that $\mathrm{NAD}(\mathrm{P}) \mathrm{H}$ fluorescence lifetime differences observed between healthy and pathological states reflect shifts in the NADPH/NADH balance.

In this work, termination of oxidative phosphorylation by uncoupling and of glycolysis by glucose deprivation could not be resolved on the basis of the fluorescence decay parameters measured under these conditions. In addition, two of the cell lines studied here with similar fluorescence decay parameters were shown to differ in their reliance on aerobic and anaerobic metabolism (wild-type HEK293 and NADK - ). This implies that changes in the NAD $(\mathrm{P}) \mathrm{H}$ fluorescence decay do not simply report shifts between an oxidative or glycolytic phenotype, but reflect the 
differential response of the NAD and NADP pools following a metabolic alteration. For example, both uncoupling and electron transport chain inhibition terminate aerobic ATP production yet have opposing effects on the overall $\mathrm{NAD}(\mathrm{P})$ redox state and thus, perhaps on $\alpha_{\text {bound }}$. Changes in $\tau_{\text {bound }}$ will be induced by metabolic transitions that cause divergent effects on NAD- and NADP-associated pathways. Thus, neither parameter alone will permit the detection of oxidative or glycolytic states using $\mathrm{NAD}(\mathrm{P}) \mathrm{H}$ fluorescence decay measurements. However, a method for assessing the pathways used for ATP production by measuring autofluorescence may be possible by combining a number of observables, including $\alpha_{\text {bound }}$ and $\tau_{\text {bound, }}$, alongside fluorescence intensity information and similar measurements from flavoprotein fluorescence as part of a spectrally resolved lifetime imaging tool ${ }^{24}$.

In the present study, we have investigated the canonical form of FLIM in which fluorescence lifetimes $\tau_{\text {free }}$ and $\tau_{\text {bound }}$, along with the weighting parameter $\alpha_{\text {bound, }}$ are extracted by leastsquares fitting of a biexponential decay at each pixel ${ }^{11,24-26}$. Drawbacks of this method of analysis have been identified, such as the computational burden of fitting $10^{4}-10^{5}$ independent decay curves in a single image, difficulties in resolving decay components with closely spaced lifetimes and correlations between the fit parameters ${ }^{2}$. Other groups have therefore focussed on developing novel analytical techniques. For example, Yaseen et al. ${ }^{53}$ recently applied global analysis to the application of NAD(P)H FLIM of the rat cortex. In this approach, the lifetimes of four decay components were shared across the image and a novel computational algorithm recovered the optimum amplitudes of each species to describe the fluorescence decay at each pixel. Interestingly, two enzyme-bound components were identified in the tissue with lifetimes of 1.7 and $3.2 \mathrm{~ns}$, perhaps corresponding to enzyme-bound NADH and NADPH. Another approach to which our conclusions may be strongly applicable is the phasor method developed by Digman et al..$^{52}$ Here, the real and imaginary parts of the Fourier transform of the fluorescence decay at each pixel of a FLIM image define the coordinates of a point in a two-dimensional phase space. The relative abundance of two or more fluorescent species with different lifetimes can then be inferred from the location of the pixels in the phasor plot. Application of this 'fit-free' approach to separating NADH and NADPH fluorescence will be the subject of further work.

Altogether, we have shown that the fluorescence lifetime characteristics of $\mathrm{NAD}(\mathrm{P}) \mathrm{H}$ in live cells and tissues can be used to discriminate between NADH and NADPH fluorescence, providing, for the first time, a biochemical framework for interpretation of $\mathrm{NAD}(\mathrm{P}) \mathrm{H}$ FLIM studies. Such a technique permits the separation of NADH and NADPH redox signalling without disrupting the sample on the addition of external probes ${ }^{54,55}$, allowing complex tissue preparations to be investigated. The approach revealed previously unknown cellular metabolic specializations in the mammalian cochlea, highlighting a subpopulation of cells characterized by high NADPH levels, opening up new avenues of research to understand the functional significance of redox pathways with respect to the physiological roles of these cells.

\section{Methods}

Cell culture. HEK293 cells were obtained from the American Type Culture Collection and grown in advanced Dulbecco's modified Eagle Medium (Gibco) supplemented with $10 \%$ fetal bovine serum, $2 \mathrm{mM}$ GlutaMAX, $100 \mathrm{U} \mathrm{ml}^{-1}$ penicillin and $100 \mu \mathrm{g} \mathrm{ml}^{-1}$ streptomycin (Gibco). Production of the NADK + and NADK - cell lines has been reported previously ${ }^{29}$. These cultures were additionally supplemented with $0.1 \mathrm{mg} \mathrm{ml}^{-1} \mathrm{G}-418$ selective antibiotic (Gibco). All cells were grown as monolayers in sterile $75 \mathrm{~cm}^{2}$ tissue culture flasks (Thermo Scientific Nunc) in a $37^{\circ} \mathrm{C}, 5 \% \mathrm{CO}_{2}$ incubator.
Cochlea explant cultures. Cochlear coils were isolated from male and female post-natal day 2-3 Sprague Dawley rats as previously described ${ }^{56}$. Briefly, auditory bullae were removed and transferred into Medium 199 with Hank's balanced salts (Life Technologies). The cartilaginous wall of the bulla was opened and the whole cochlea extracted. The stria vascularis and Reissner's membrane were removed and the cochlea cut into three coils. The cochlear coils were placed onto Cell-Tak cell and tissue adhesive (BD Biosciences)-coated dishes (MatTek). For coating, cell adhesive was diluted to $70 \mu \mathrm{g} \mathrm{ml}^{-1}$ in $0.1 \mathrm{mM} \mathrm{NaHCO}_{3}$. The cochlear explants were incubated overnight in DMEM/F12 (Gibco), supplemented with 1\% fetal bovine serum (Life Technologies) in a $37^{\circ} \mathrm{C}, 5 \% \mathrm{CO}_{2}$ incubator. The isolation was performed in accordance with the United Kingdom Animals (Scientific Procedures) Act of 1986 and in compliance with the Biological Services Management Group and the Biological Services Ethical Committee, University College London.

Live-cell microscopy. On the microscope stage, coverslips containing 300,000 cells were maintained at $37^{\circ} \mathrm{C}$ in a metal ring and bathed in DMEM solution at $\mathrm{pH} 7.4$ containing $10 \mathrm{mM}$ HEPES and $2 \mathrm{mM}$ GlutaMAX. Glucose $(25 \mathrm{mM})$ was present under control conditions and during EGCG treatment $(100 \mu \mathrm{M}$ in cell lines, $200 \mu \mathrm{M}$ for cochlea). Pharmacological perturbations to metabolism were applied by the dropwise addition of working concentrations of each compound, diluted from stock solutions in DMEM recording medium.

NAD(P)H FLIM was performed on an upright LSM 510 microscope (Carl Zeiss) with a $1.0 \mathrm{NA} \times 40$ water-dipping objective using a 650 -nm short-pass dichroic and $460 \pm 25 \mathrm{~nm}$ emission filter. Two-photon excitation was provided by a Chameleon (Coherent) Ti:sapphire laser tuned to $700 \mathrm{~nm}$, with on-sample powers kept below $10 \mathrm{~mW}$. Spectral controls (see Supplementary Fig. 4 and Supplementary Note 5) confirmed the NAD $(\mathrm{P}) \mathrm{H}$ specificity of this excitation wavelength and emission filtering. Photodamage controls (see Supplementary Tables 6 and 7 and Supplementary Note 6) demonstrated that FLIM parameters were not varying over the course of imaging. Emission events were registered by an external detector (HPM-100, Becker \& Hickl) attached to a commercial time-correlated single photon counting electronics module (SPC-830, Becker \& Hickl) contained on a PCI board in a desktop computer. Scanning was performed continuously for $4 \mathrm{~min}$ with a pixel dwell time of $1.6 \mu$ s. To identify mitochondrial and cytosolic regions, the mitochondrially targeted fluorescent dye tetramethylrhodamine methyl ester (TMRM) was added to the recording medium, at a final concentration of $25 \mathrm{nM}$, $20 \mathrm{~min}$ before imaging. TMRM fluorescence was collected for a 10-s burst using a $610 \pm 30 \mathrm{~nm}$ emission filter. Excitation was provided at the same wavelength as $\mathrm{NAD}(\mathrm{P}) \mathrm{H}$ to avoid possible chromatic aberration. The $585 \pm 15 \mathrm{~nm}$ emission spectrum of TMRM ensured its fluorescence did not contaminate the NAD $(\mathrm{P}) \mathrm{H}$ FLIM images.

$\mathrm{NAD}(\mathrm{P}) \mathrm{H}$ fluorescence intensity time series and MCB imaging were performed on an inverted LSM 510 laser scanning confocal microscope (Carl Zeiss) with $351 \mathrm{~nm}$ illumination from an argon ion laser (Coherent Enterprise UV). MCB $(30$ min loading at $50 \mu \mathrm{M})$ and $\mathrm{NAD}(\mathrm{P}) \mathrm{H}$ fluorescence were observed using a 351-nm long-pass dichroic and $460 \pm 25 \mathrm{~nm}$ band-pass emission filter with a $\times 40$, 1.3 NA quartz oil immersion objective. Images $(12$-bit $512 \times 512)$ were obtained with a pixel dwell time of $1.6 \mu \mathrm{s}$. Time series measurements were obtained at $1 \mathrm{~min}$ intervals. To reduce noise, the image recorded at each time point was a mean of four consecutive scans. Fluorescence intensity levels were extracted using ImageJ (NIH).

Metabolic controls. For the measurement of oxygen consumption, cells were trypsinized and resuspended at $\sim 1$ million cells per $\mathrm{ml}$ in DMEM buffered with $10 \mathrm{mM}$ HEPES and supplemented with $25 \mathrm{mM}$ glucose and $2 \mathrm{mM}$ GlutaMAX. Respiration rates were measured in triplicate at $37^{\circ} \mathrm{C}$ with the high-resolution Oxygraph (Oroboros Oxygraph-2k). State 4 respiration values were obtained in the presence of $2.5 \mu \mathrm{M}$ oligomycin, maximal oxidative capacities were determined in the presence of $2 \mu \mathrm{M}$ FCCP and non-mitochondrial background oxygen consumption was determined in the presence of $2.5 \mu \mathrm{M}$ antimycin A. Lactate release rates were measured in triplicate by removing a sample of serum-free DMEM from $70 \%$ confluent cell cultures at $1 \mathrm{~h}$ intervals for $5 \mathrm{~h}$ total and determining the concentration of lactate present using a commercially available plate-reader assay (Sigma Aldrich) in absorption mode. For normalization of both oxygen consumption and lactate release rates, final cell counts were performed using a haemocytometer.

FLIM data analysis. Following $5 \times 5$ binning of photon counts at each pixel, fluorescence decay curves of the form

$$
I(t)=Z+I_{0}\left[\left(1-\alpha_{\text {bound }}\right) \exp \left(-\frac{t}{\tau_{\text {free }}}\right)+\alpha_{\text {bound }} \exp \left(-\frac{t}{\tau_{\text {bound }}}\right)\right]
$$

were fit to the FLIM images using iterative reconvolution in SPCImage (Becker \& Hickl), where $Z$ allows for time-uncorrelated background noise. The instrument response function of the FLIM system was obtained by measuring the fluorescence decay profile of second harmonic generation by a potassium dihydrogen phosphate (KDP) crystal at $920 \mathrm{~nm}$, grown by leaving a molar solution of KDP in water on a coverslip to evaporate. Matrices of the fit parameters $\tau_{\text {free }}, \alpha_{\text {bound }}$ and $\tau_{\text {bound }}$, along 
with the total photons counted at each pixel, were exported from SPCImage. In MATLAB (The Mathworks), a 16-bit grayscale image was produced for each parameter matrix in which the intensity of each pixel was proportional to the parameter value at that location. ImageJ was then used to measure the grayscale intensity in the parameter images and the values obtained converted back to parameter values using the scaling factors applied in their production. Masks identifying the location of mitochondrial and cytosolic pixels were created by importing images of the TMRM distribution in the cell and a nuclear mask was defined by hand, allowing the parameters describing the fluorescence decay of $\mathrm{NAD}(\mathrm{P}) \mathrm{H}$ in each of these regions to be extracted separately (Supplementary Fig. 2a).

Statistical analysis. $\mathrm{NAD}(\mathrm{P}) \mathrm{H}$ fluorescence lifetime parameters obtained using FLIM are reported as a mean over at least three regions of at least three separate cultures. Uncertainties in these values were taken as the s.d. of the measurements. Differences between the lifetime parameters measured under different conditions were tested for statistical significance $(P<0.05)$ using a two-tailed Student's $t$-test. Statistically significant differences between data sets normalized to be expressed in relative arbitrary units (fluorescence intensity, NADH/NADPH concentration) were assessed using a Wilcoxon signed-rank test. For the estimation of uncertainties in the predictions of the numerical model, the standard formula for the calculation of the error $\sigma_{z}^{2}$ in an arbitrary function $Z=Z(A, B, C, \ldots)$ was applied,

$$
\sigma_{z}^{2}=\left(\frac{\partial Z}{\partial A}\right)^{2} \sigma_{A}^{2}+\left(\frac{\partial Z}{\partial B}\right)^{2} \sigma_{B}^{2}+\left(\frac{\partial Z}{\partial C}\right)^{2} \sigma_{C}^{2} \cdots
$$

\section{References}

1. Collins, Y. et al. Mitochondrial redox signalling at a glance. J. Cell Sci. 125, 801-806 (2012).

2. Ying, W. NAD + /NADH and NADP +/NADPH in cellular functions and cell death: regulation and biological consequences. Antioxid. Redox Signal. 10, 179-206 (2008).

3. Mailloux, R., Lemire, J. \& Appanna, V. Metabolic networks to combat oxidative stress in Pseudomonas fluorescens. Antonie Van Leeuwenhoek 99, 433-442 (2011).

4. Rocheleau, J. V., Head, W. S. \& Piston, D. W. Quantitative NAD(P)H/ flavoprotein autofluorescence imaging reveals metabolic mechanisms of pancreatic islet pyruvate response. J. Biol. Chem. 279, 31780-31787 (2004).

5. Chance, B., Schoener, B., Oshino, R., Itshak, F. \& Nakase, Y. Oxidationreduction ratio studies of mitochondria in freeze-trapped samples. NADH and flavoprotein fluorescence signals. J. Biol. Chem. 254, 4764-4771 (1979).

6. Patterson, G. H., Knobel, S. M., Arkhammar, P., Thastrup, O. \& Piston, D. W. Separation of the glucose-stimulated cytoplasmic and mitochondrial NAD $(\mathrm{P}) \mathrm{H}$ responses in pancreatic islet beta cells. Proc. Natl Acad. Sci. USA 97, 5203-5207 (2000).

7. De Ruyck, J. et al. Towards the understanding of the absorption spectra of $\mathrm{NAD}(\mathrm{P}) \mathrm{H} / \mathrm{NAD}(\mathrm{P})+$ as a common indicator of dehydrogenase enzymatic activity. Chem. Phys. Lett. 450, 119-122 (2007).

8. Duchen, M. R., Surin, A. \& Jacobson, J. Imaging mitochondrial function in intact cells. Methods Enzymol. 361, 353-389 (2003).

9. Spat, A. \& Pitter, J. G. The effect of cytoplasmic Ca2 + signal on the redox state of mitochondrial pyridine nucleotides. Mol. Cell. Endocrinol. 215, 115-118 (2004).

10. Vishwasrao, H. D., Heikal, A. A., Kasischke, K. A. \& Webb, W. W. Conformational dependence of intracellular NADH on metabolic state revealed by associated fluorescence anisotropy. J. Biol. Chem. 280, 25119-25126 (2005).

11. Skala, M. C. et al. In vivo multiphoton fluorescence lifetime imaging of proteinbound and free nicotinamide adenine dinucleotide in normal and precancerous epithelia. J. Biomed. Opt. 12, 24014 (2007).

12. Yu, Q. \& Heikal, A. A. Two-photon autofluorescence dynamics imaging reveals sensitivity of intracellular NADH concentration and conformation to cell physiology at the single-cell level. J. Photochem. Photobiol. B 95, 46-57 (2009).

13. Evans, N. D., Gnudi, L., Rolinski, O. J., Birch, D. J. S. \& Pickup, J. C. Glucosedependent changes in $\mathrm{NAD}(\mathrm{P}) \mathrm{H}$-related fluorescence lifetime of adipocytes and fibroblasts in vitro: potential for non-invasive glucose sensing in diabetes mellitus. J. Photochem. Photobiol. B 80, 122-129 (2005).

14. Butte, P. V. et al. Intraoperative delineation of primary brain tumors using time-resolved fluorescence spectroscopy. J. Biomed. Opt. 15, 27008 (2010).

15. Sanchez, W. Y., Prow, T. W., Sanchez, W. H., Grice, J. E. \& Roberts, M. S. Analysis of the metabolic deterioration of ex vivo skin from ischemic necrosis through the imaging of intracellular $\mathrm{NAD}(\mathrm{P}) \mathrm{H}$ by multiphoton tomography and fluorescence lifetime imaging microscopy. J. Biomed. Opt. 15, 46008-46011 (2010).

16. Yu, J.-S., Guo, H.-W., Wang, C.-H., Wei, Y.-H. \& Wang, H.-W. Increase of reduced nicotinamide adenine dinucleotide fluorescence lifetime precedes mitochondrial dysfunction in staurosporine-induced apoptosis of HeLa cells. J. Biomed. Opt. 16, 36008 (2011).
17. Gehlsen, U. et al. Two-photon fluorescence lifetime imaging monitors metabolic changes during wound healing of corneal epithelial cells in vitro. Graefes Arch. Clin. Exp. Ophthalmol. 250, 1293-1302 (2012).

18. Stringari, C. et al. Metabolic trajectory of cellular differentiation in small intestine by Phasor Fluorescence Lifetime Microscopy of NADH. Sci. Rep. 2, 568 (2012).

19. Blacker, T. S., Marsh, R. J., Duchen, M. R. \& Bain, A. J. Activated barrier crossing dynamics in the non-radiative decay of NADH and NADPH. Chem. Phys. 422, 184-194 (2013).

20. Scott, T. G., Spencer, R. D., Leonard, N. J. \& Weber, G. Synthetic spectroscopic models related to coenzymes and base pairs. V. Emission properties of NADH. Studies of fluorescence lifetimes and quantum efficiencies of NADH, AcPyADH, [reduced acetylpyridineadenine dinucleotide] and simplified synthetic models. J. Am. Chem. Soc. 92, 687-695 (1970).

21. Gafni, A. \& Brand, L. Fluorescence decay studies of reduced nicotinamide adenine dinucleotide in solution and bound to liver alcohol dehydrogenase. Biochemistry 15, 3165-3171 (1976).

22. Wang, H. W. et al. Differentiation of apoptosis from necrosis by dynamic changes of reduced nicotinamide adenine dinucleotide fluorescence lifetime in live cells. J. Biomed. Opt. 13, 054011 (2008).

23. Zheng, W., Li, D. \& Qu, J. Y. Monitoring changes of cellular metabolism and microviscosity in vitro based on time-resolved endogenous fluorescence and its anisotropy decay dynamics. J. Biomed. Opt. 15, 37011-37013 (2010).

24. Skala, M. C. et al. In vivo multiphoton microscopy of NADH and FAD redox states, fluorescence lifetimes, and cellular morphology in precancerous epithelia. Proc. Natl Acad. Sci. USA 104, 19494-19499 (2007).

25. Bird, D. K. et al. Metabolic mapping of MCF10A human breast cells via multiphoton fluorescence lifetime imaging of the coenzyme NADH. Cancer Res. 65, 8766-8773 (2005)

26. Pradhan, A. et al. Steady state and time-resolved fluorescence properties of metastatic and non-metastatic malignant cells from different species. J. Photochem. Photobiol. B 31, 101-112 (1995).

27. Grinvald, A. \& Steinberg, I. Z. On the analysis of fluorescence decay kinetics by the method of least-squares. Anal. Biochem. 59, 583-598 (1974).

28. Scrutton, N. S., Berry, A. \& Perham, R. N. Redesign of the coenzyme specificity of a dehydrogenase by protein engineering. Nature 343, 38-43 (1990).

29. Pollak, N., Niere, M. \& Ziegler, M. NAD kinase levels control the NADPH concentration in human cells. J. Biol. Chem. 282, 33562-33571 (2007).

30. Shin, E. S. et al. Catechin gallates are NADP +-competitive inhibitors of glucose-6-phosphate dehydrogenase and other enzymes that employ NADP + as a coenzyme. Bioorg. Med. Chem. 16, 3580-3586 (2008).

31. Schomburg, I. et al. BRENDA: a resource for enzyme data and metabolic information. Trends Biochem. Sci. 27, 54-56 (2002).

32. Huxley, L., Quirk, P. G., Cotton, N. P. J., White, S. A. \& Jackson, J. B. The specificity of proton-translocating transhydrogenase for nicotinamide nucleotides. Biochim. Biophys. Acta 1807, 85-94 (2011).

33. Enander, K. \& Rydstrom, J. Energy-linked nicotinamide nucleotide transhydrogenase. Kinetics and regulation of purified and reconstituted transhydrogenase from beef heart mitochondria. J. Biol. Chem. 257, 14760-14766 (1982).

34. Klaidman, L. K., Leung, A. C. \& Adams, J. D. High-performance liquid chromatography analysis of oxidized and reduced pyridine dinucleotides in specific brain regions. Anal. Biochem. 228, 312-317 (1995).

35. Keelan, J., Allen, N. J., Antcliffe, D., Pal, S. \& Duchen, M. R. Quantitative imaging of glutathione in hippocampal neurons and glia in culture using monochlorobimane. J. Neurosci. Res. 66, 873-884 (2001).

36. Rossmann, M. G., Moras, D. \& Olsen, K. W. Chemical and biological evolution of a nucleotide-binding protein. Nature 250, 194-199 (1974).

37. Carugo, O. \& Argos, P. NADP-dependent enzymes. I: conserved stereochemistry of cofactor binding. Proteins 28, 10-28 (1997).

38. Niesner, R. et al. Selective detection of NADPH oxidase in polymorphonuclear cells by means of $\mathrm{NAD}(\mathrm{P}) \mathrm{H}$-based fluorescence lifetime imaging. J. Biophys. 2008, 602639 (2008).

39. Kolker, E. et al. MOPED: Model Organism Protein Expression Database. Nucleic Acids Res. 40, D1093-D1099 (2012).

40. Rebhan, M., Chalifa-Caspi, V., Prilusky, J. \& Lancet, D. GeneCards: integrating information about genes, proteins and diseases. Trends Genet. 13, 163 (1997).

41. Shatwell, K. P. \& Segal, A. W. NADPH oxidase. Int. J. Biochem. Cell Biol. 28, 1191-1195 (1996).

42. Iyer, G. Y. N., Islam, M. F. \& Quastel, J. H. Biochemical aspects of phagocytosis. Nature 192, 535-541 (1961).

43. Tadrous, P. J. et al. Fluorescence lifetime imaging of unstained tissues: early results in human breast cancer. J. Pathol. 199, 309-317 (2003).

44. Sud, D., Zhong, W., Beer, D. G. \& Mycek, M. A. Time-resolved optical imaging provides a molecular snapshot of altered metabolic function in living human cancer cell models. Opt. Express 14, 4412-4426 (2006).

45. Colasanti, A. et al. MS-2 fibrosarcoma characterization by laser induced autofluorescence. Lasers Surg. Med. 26, 441-448 (2000). 
46. Butte, P. V. et al. Diagnosis of meningioma by time-resolved fluorescence spectroscopy. J. Biomed. Opt. 10, 64026-64029 (2005).

47. De Beule, P. A. et al. A hyperspectral fluorescence lifetime probe for skin cancer diagnosis. Rev. Sci. Instrum. 78, 123101 (2007).

48. Sun, Y. et al. Fluorescence lifetime imaging microscopy: in vivo application to diagnosis of oral carcinoma. Opt. Lett. 34, 2081-2083 (2009).

49. Sun, Y. et al. Fluorescence lifetime imaging microscopy for brain tumor imageguided surgery. J. Biomed. Opt. 15, 56022 (2010).

50. McGinty, J. et al. Wide-field fluorescence lifetime imaging of cancer. Biomed. Opt. Express 1, 627-640 (2010).

51. Vander Heiden, M. G., Cantley, L. C. \& Thompson, C. B. Understanding the Warburg effect: the metabolic requirements of cell proliferation. Science 324, 1029-1033 (2009).

52. Digman, M. A., Caiolfa, V. R., Zamai, M. \& Gratton, E. The phasor approach to fluorescence lifetime imaging analysis. Biophys. J. 94, L14-L16 (2008).

53. Yaseen, M. A. et al. In vivo imaging of cerebral energy metabolism with two-photon fluorescence lifetime microscopy of NADH. Biomed. Opt. Express 4, 307-321 (2013).

54. Zhao, Y. et al. Genetically encoded fluorescent sensors for intracellular NADH detection. Cell Metab. 14, 555-566 (2011).

55. Hung, Y. P., Albeck, J. G., Tantama, M. \& Yellen, G. Imaging cytosolic NADH-NAD + redox state with a genetically encoded fluorescent biosensor. Cell Metab. 14, 545-554 (2011).

56. Mann, Z. F., Duchen, M. R. \& Gale, J. E. Mitochondria modulate the spatio-temporal properties of intra- and intercellular $\mathrm{Ca} 2+$ signals in cochlear supporting cells. Cell Calcium 46, 136-146 (2009).

\section{Acknowledgements}

We thank Dr Will Kotiadis for assistance with respirometry measurements, Dr Zhi Yao for assistance with the lactate release assay and Dr Laura Osellame for proofreading of the manuscript. Action on Hearing Loss supported the work of Z.F.M. and J.E.G.
G.S. was funded by Parkinson's UK, British Heart Foundation, Wellcome Trust-UCL Therapeutic Innovation Fund, Telethon (Italy, GEP1206) and the Italian Association for Cancer Research (AIRC). M.R.D. acknowledges strategic funding from the Wellcome Trust/MRC Joint Call in Neurodegeneration Award (WT089698). T.S.B. is grateful for the award of an EPSRC studentship and the inaugural Professor Anne Warner postdoctoral fellowship through the CoMPLEX Doctoral Training Centre at UCL.

\section{Authors contributions}

M.Z. provided the NADK + and NADK- cell lines. J.E.G. prepared cochlea cultures and oversaw their imaging. Z.F.M., G.S. and M.R.D. performed preliminary experiments T.S.B. performed the experiments, data analysis and modelling. A.J.B., G.S. and M.R.D. supervised the work. T.S.B., G.S. and M.R.D. wrote the manuscript.

\section{Additional information}

Supplementary Information accompanies this paper at http://www.nature.com/ naturecommunications

Competing financial interests: The authors declare no competing financial interests

Reprints and permission information is available online at http://npg.nature.com/ reprintsandpermissions/

How to cite this article: Blacker, T. S. et al. Separating NADH and NADPH fluorescence in live cells and tissues using FLIM. Nat. Commun. 5:3936 doi: 10.1038/ncomms4936 (2014).

(c) (i) This work is licensed under a Creative Commons Attribution 3.0 Unported License. The images or other third party material in this article are included in the article's Creative Commons license, unless indicated otherwise in the credit line; if the material is not included under the Creative Commons license, users will need to obtain permission from the license holder to reproduce the material. To view a copy of this license, visit http://creativecommons.org/licenses/by/3.0/ 